\title{
Serological Study of Human Toxocariasis Using Elisa Technique in Six Hospitals, Adamawa State, Nigeria
}

\author{
Terry Thomas ${ }^{1 *} \quad$ Qadeer Mohammed $^{2} \quad$ Pukuma Micah $^{2}$ \\ 1.Department of Biomedical Sciences and Technology, Federal Polytechnic P.M.B 35, Mubi, Adamawa state, \\ Nigeria \\ 2.Department of Zoology, School of Life Sciences, Modibbo Adama University of Technology, Yola, Adamawa
} State, Nigeria

\begin{abstract}
Study on the Human Seroprevalence of Toxocara canis (T.canis) Antibody titre using ELISA Technique was conducted in Six Hospitals of Adamawa State Nigeria, between the months of January to June 2018. Five hundred and sixty four (564) samples were collected using non randomised sampling technique. Statistical analyses were carried out using Statistix 9.1 and the results obtained from the study revealed an overall Seroprevalence rate of $63.65 \%$. Age range 21 to 30 years indicated that $7.45 \%$ Males and $13.65 \%$ Females were positive, with age showing no any significant correlation to Anti-Toxocara Antibody titre. Also results based on Sex revealed that $215(38.12 \%)$ Females and $144(25.53 \%)$ Males reacted positive with Sex having no significant correlation to Anti-Toxocara Antibody titre. Seroprevalence results based on Religion and Dog ownership indicated that 167(29.61\%) Christians and 97(17.21\%) Moslems were positive with Religion showing a negative correlation. Owners of dogs accounted for 58(10.28\%) of the samples analysed, with dog ownership showing a negative correlation. Seropositivity base on Socioeconomic Status, reveals that Housewives had $141(25.01 \%)$ while under care (children under one year) had 23(4.08\%) with socioeconomic status showing an association with Anti-Toxocara Antibody titre. Results on Tribes reveals that Fulani's had 67(11.85\%), Yalla accounted for $1(0.18 \%)$ with Tribe showing a weak significance difference to Anti- Toxocara Antibody titre. In conclusion the study showed that there is a very high Seroprevalence of Toxocara canis Antibody titre amongst the studied population. Therefore it is recommended that Anti-Toxocara Antibody screening be included into routine medical diagnosis to also cover private medical outfits. It is also recommended that further studies on molecular diagnosis be carried out to ascertain the gene markers responsible for the pathology caused to the host by (T.canis).
\end{abstract}

Keywords: Antibody, Seroprevalence, Religion, Tribe, Adamawa, pork, Toxocara canis and Seropositivity DOI: $10.7176 / \mathrm{JBAH} / 9-10-06$

Publication date:May $31^{\text {st }} 2019$

\section{Introduction}

Toxocariasis is a neglected infection that has a worldwide distribution. T.canis is the most relevant agent due to its frequent occurrence in humans. Over the years, this disease has drawn much attention because of its surprisingly high prevalence (Anna et al,. 2018). Parasite eggs are commonly found in soil and canine fur, dirty hands, consumption of vegetables contaminated with embryonated eggs or consumption of embryonated egg in paratenic hosts such as chicken, beef from cattle, pork and other small animals. Previous studies have reported varying seroprevalence rates in different locations which agree with the findings in this study. The overall seroprevalence rate in this study was $63.63 \%$ though higher agrees with similar work earlier conducted by Alonso et al,. (2000), who reported a positivity of 37.9\% in Argentina; Epinoza et al,. (2008) reported 32.4\% positivity in Peru and Brazil. Similarly, Ajayi et al,. (2014) reported $29.8 \%$ positivity in Jos, Nigeria. Other findings show $1.6 \%$ in Japan, 2.4\% in Denmark, $6.3 \%$ in Australia, $7 \%$ in Sweden, $14 \%$ in USA and $19.6 \%$ in Malaysia (Guangxu et al,. 2018).

\section{Materials and Methods \\ Study Area:}

Adamawa State is located at the North Eastern part of Nigeria. It lies between latitude $7^{0}$ and $11^{\circ} \mathrm{N}$ of the equator and between longitude $11^{\circ}$ and $14{ }^{\circ} \mathrm{E}$ of the Greenwich meridian. It shares boundary with Taraba State in the South and West, Gombe State in the Northwest and Borno to the North. Adamawa State has an international boundary with the Cameroon Republic along the Eastern border. The State covers a land area of about $38,741 \mathrm{~km}$ with a population of about 2,102,053 people according to the 1991 census. Adamawa State is divided into 21 Local Government Areas Fig 3.1 (Adebayo and Tukur 1999). 


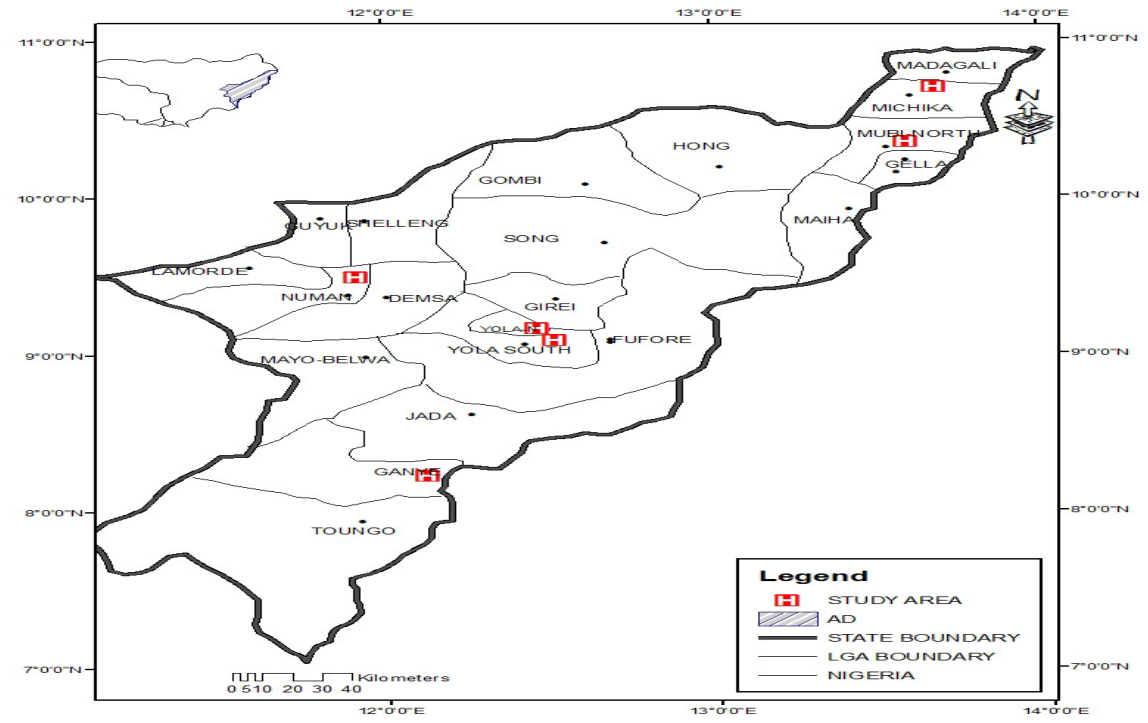

Fig1. Map of Adamawa State Showing Study Sites Data collection

Data were collected through a designed data collection form to capture information and through blood samples from patients' refereed to the Haematology Laboratories of the various Hospitals in the study area.

\section{Study design and Sample collection}

One hundred (100) blood samples each were collected from the six Hospitals in the study area. A non randomised sampling technique were used, where whole blood was collected through the venous puncture with the aid of Sterile syringe and approximately $5 \mathrm{ml}$ of whole blood was obtained and emptied into plain gel vaccontainer tubes pre-labelled with information on Age, Sex, Location, Socioeconomic Status, Tribe, Religion and whether the patient's own dog or not. The blood samples were than centrifuged to separate serum from the red cells, the serums were then used for analysis.

\section{Laboratory Analysis}

Enzyme Link Immunoabsorbent Assay (ELISA) Toxocara canis Antigen Kit product №.8206-35 supplied by Diagnosis Automation/Cortez Diagnostics Inc. Woodland Hills California USA was used for Analysis which were carried out in the Haematology Laboratory of the Federal Medical Centre Yola, Adamawa State Nigeria.

\section{Statistical analysis:}

Statistix 9.1 (2012) Statistical package for scientist and engineers, USA, was used for statistical analysis.

\section{RESULTS/ DISCUSSION}

Results obtained from the study based on hospital location figure 2 shows that State Specialist Hospital, Yola accounted for 76(13.49\%), Numan 75(13.29\%), Ganye 74(13.12) and Federal Medical Centre 30(5.32\%). State Specialist Hospital having a higher rate can be explained to the fact that, being a referral hospital for medical cases from other General Hospital across the State, large number of patients from different socioeconomic background visits the hospital hence patients with different medical cases are brought into the Hospital. Another reason could be that the State Specialist Hospital being located in the State capital allows many patients to have access despite not been referred there by other hospitals. The Federal Medical Centre being a referral hospital also handles only special medical cases, thereby less number of patients visit the Centre as compared with the later. The results further indicate that seroprevalence rates were high amongst rural hospitals than urban as the results obtained from Ganye (13.12\%) and Numan (13.29\%) shows higher rates when compared with Federal Medical Centre Yola (5.32\%). This finding agrees with earlier result reported by Chiodo et al,. (2006), which indicates that rural populace have higher rates of infection with T.canis than those in the urban areas. This finding maybe in part due to differences in level of personal and community hygiene or cultural habits amongst different communities. Statistically the result obtained from this study revealed that, hospital location though having a negative correlation $(\mathrm{r}-0.1638)$ is highly significant $(\mathrm{P}=0.0001)$ to the risk of infection with Toxocara canis Antibody. 


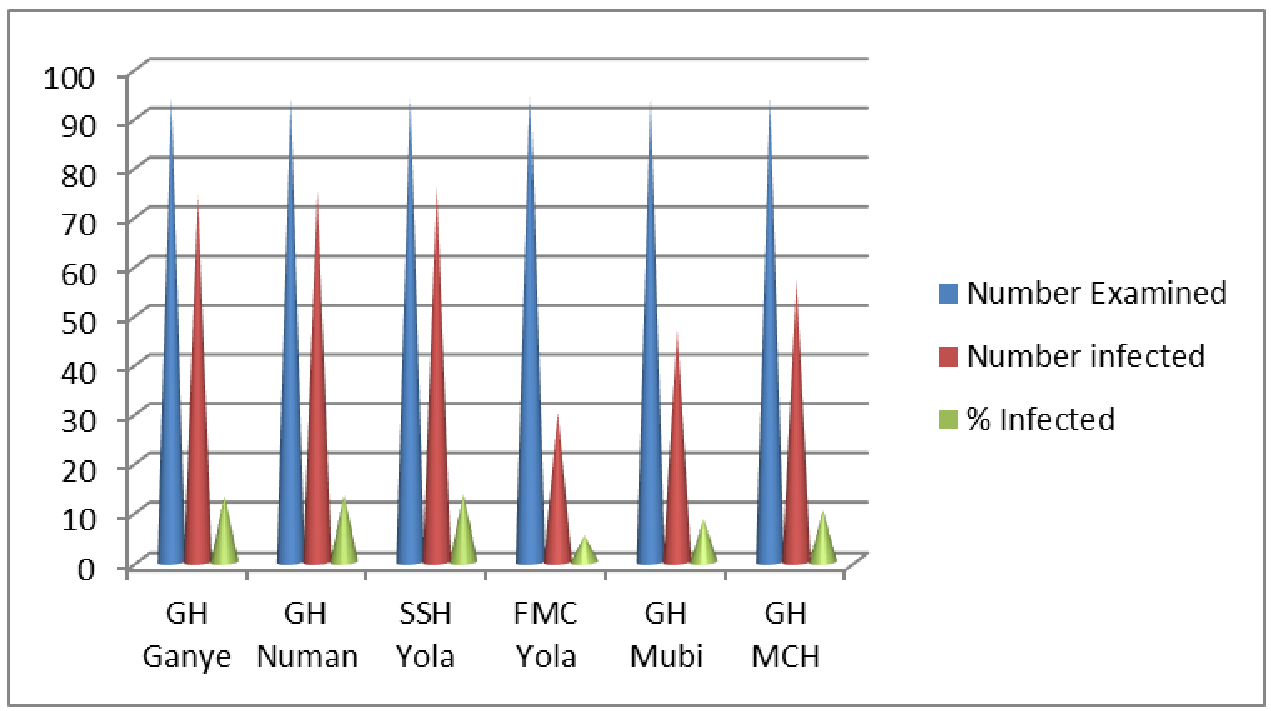

Figure2. Seroprevalence of Anti-Toxocara Antibody titre based on location

The seroprevalence based on sex Table 3 revealed that Males had (25.53\%) and Females (38.3\%). The findings in this study shows that females have higher prevalence which is contrary to the findings of Yakubu and Audu (2009), who reported that males were more infected than females, in a study on the seroprevalence of human Toxocara canis infection in Vom, Plateau State. Statistical analysis indicated that sex had a negative correlation ( $\mathrm{r}$ ) -0.0230 , but is non-significant ( $\mathrm{P}$ 0.5850) suggesting that both sexes were equally at risk of infection with Toxocariasis. A related study in South America showed equal risk to human Toxocara infection in Male and Females (Alonso et al,. 2000; Anarum et al.2002). Females accounting for 38.3\% could be attributed to the role females play in their domestic work, such as sweeping which exposes them to canine faecal matter. Other activities include preparation of food by handling fresh vegetables, fetching and using faecally contaminated water, preparation of paratenic hosts for food and the practice of poor hand washing after engaging in farm activities as well as poor hygiene practice as earlier reported by Pius et al,. (2012).

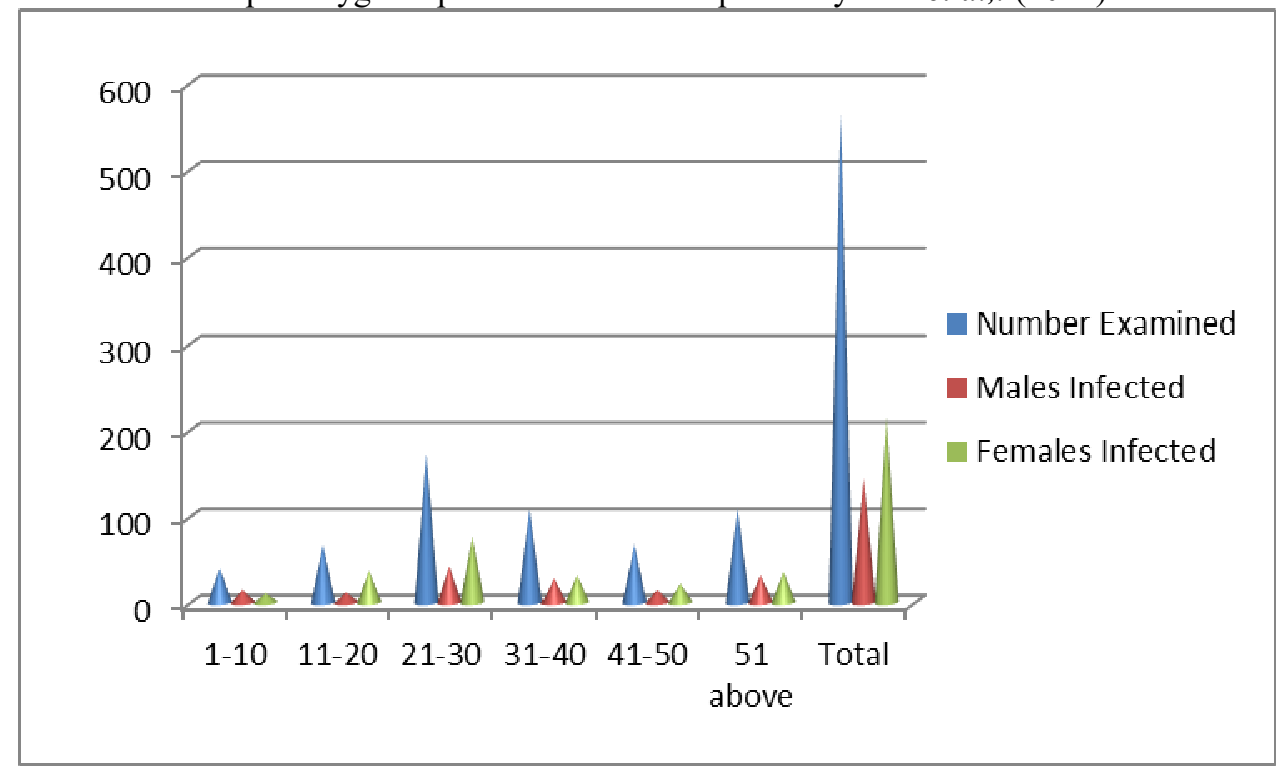

Figure3. Seroprevalence of Anti-Toxocara Antibody titre based on Age and Sex

Results based on age figure 3 indicated that age range 21-30years had the highest rate with Males $42(7.45 \%)$ and $77(13.65 \%)$ Females infected. Age range 1-10 accounted for 7.09\%, while 11-20 and 21-30 accounted for $11.87 \%$ and $30.67 \%$ respectively. This finding agrees with the work reported by Susana et al,. (2014) where it was reported that children under one year had $23.9 \%, 1$ to 2 years $42.85 \%$ and 2 years above $56 \%$. Increase in the frequency of infection with age was also observed in studies earlier reported by Mazur-Melewsk et al,. (2012); Romero-Nunez et al,. (2013) and Wisniewka-Ligier et al.. (2012) as were also found in this work. Age range of 51 and above had $109(19.32 \%)$ this can be attributed to previous exposure by those individuals to T.canis antigen after which their immune system might have developed antibodies to T.canis. as the technique used in the analysis (ELISA) does not differentiate between active infections to previous exposure of individuals 
to T.canis infection. The result obtained showed that age though having a negative correlation (r-0.0350) is non Significant $(\mathrm{P}=0.4069)$ suggesting that all ages are prone to T. canis infection in the study area

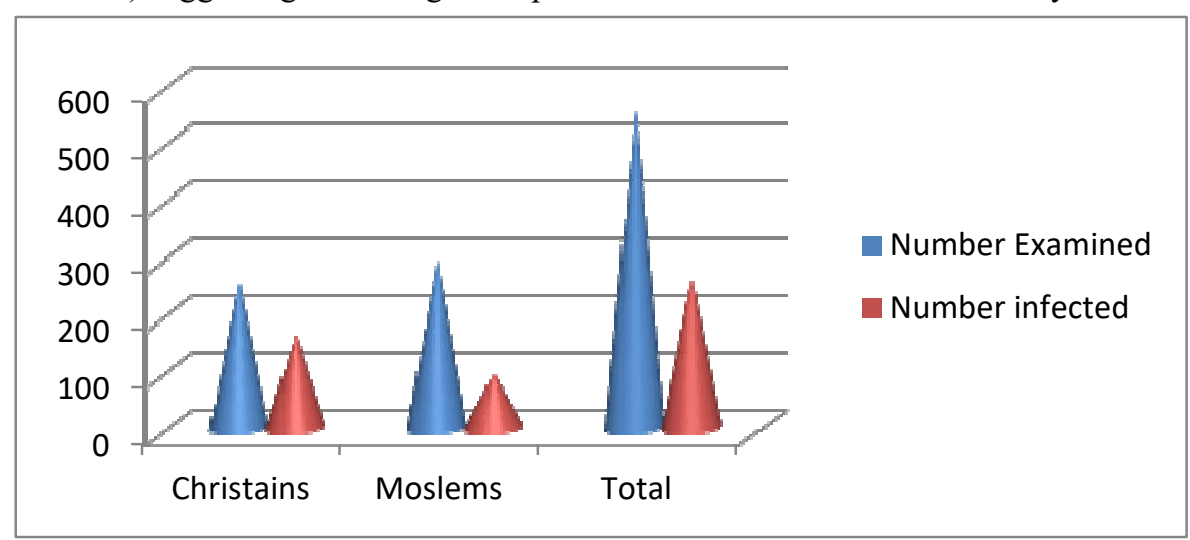

Figure4. Seroprevalence of Anti-Toxocara Antibody titre base on Religion

Different prevalence rate based on Religion figure 4 revealed that Christians accounted for 167 (29.61\%) while Moslems had 97(17.21\%) infections. Moslem accounting for lower rates of infection can be justified by their religious practices where contact with dogs is prohibited by their religious believes (Berenji et al,.2016). Tina et al,. (2016) also reported Islam forbids any contact with dog or any other material which has been in any way exposed to the animal itself or its urine and faeces. Furthermore, in Islamic countries, dogs are avoided for religious reasons, but cats are favoured as pets, the seroprevalence of human Toxocariasis can be considerable (Smith and Noordin 2006). Thus the potential roles of Toxocariasis in humans should not be ignored or underestimated (Fisher, 2003).

Christians accounting for $167(29.61 \%$ ) can be attributed to their keeping of dogs, as the religion does not prohibit the practice. Previous report have indicated that there is a significant relationship between having a dog and Toxocariasis (Nourian et al,. 2009; Berenji et al. 2016). Contact with dogs has been shown in several studies as an important risk factor for Toxocariasis. Damain et al. (2007), reported in a cross- sectional studies amongst 34 families in Amazonas State in Brazil, individuals who had contact with adult dogs, 60\% were positive, and those who had contact with puppies $66.6 \%$ demonstrating the association between contact with dogs and the presence of Toxocara Antibody. Results obtained in this work also agrees with earlier report by Chiodo et al,. (2006) who after evaluating 100 individuals for IgG anti Toxocara, 23\% were positive and all had contact with dogs. However pet owners might not be exposed to the disease more frequently than non-pet owners, especially if they take good care of their pets (Traversa et al. 2014; Gabrielli et al. 2017). Some studies refer that soil contamination is not the only effective route in human Toxocariasis and eggs of Toxocara spp. can be sprouted in fur and direct contact between humans and dogs as Aydenizoz-Ozkayhan et al,. 2008 collected 51 fur samples and observed that $21.56 \%$ of dogs had eggs on their fur. Similar studies carried out by Roddie et al 2008 and Overgaauw et al,. 2009 in the Northlands, found 12.2\% of Toxocara eggs in dog fur; moreover, many of the owners allow their dogs to climb and sleep in their beds, and only few wash their hands after contact with pets; therefore this close physical contact with pets and their owners possibly increase the risk of transmission of Toxocara spp. and point to the need for greater attention to the potential risk to which humans are exposed. Other reasons that probably accounted for Christians having a higher rate could be attributed to the role of their women in farming activities, which exposes them to contaminated soil environment which has been known to be a risk factor in T. canis infection. Religion in this study showed a negative correlation (r-0.0332) to T. canis infection and non-significant $(\mathrm{P}=0.4315)$. 


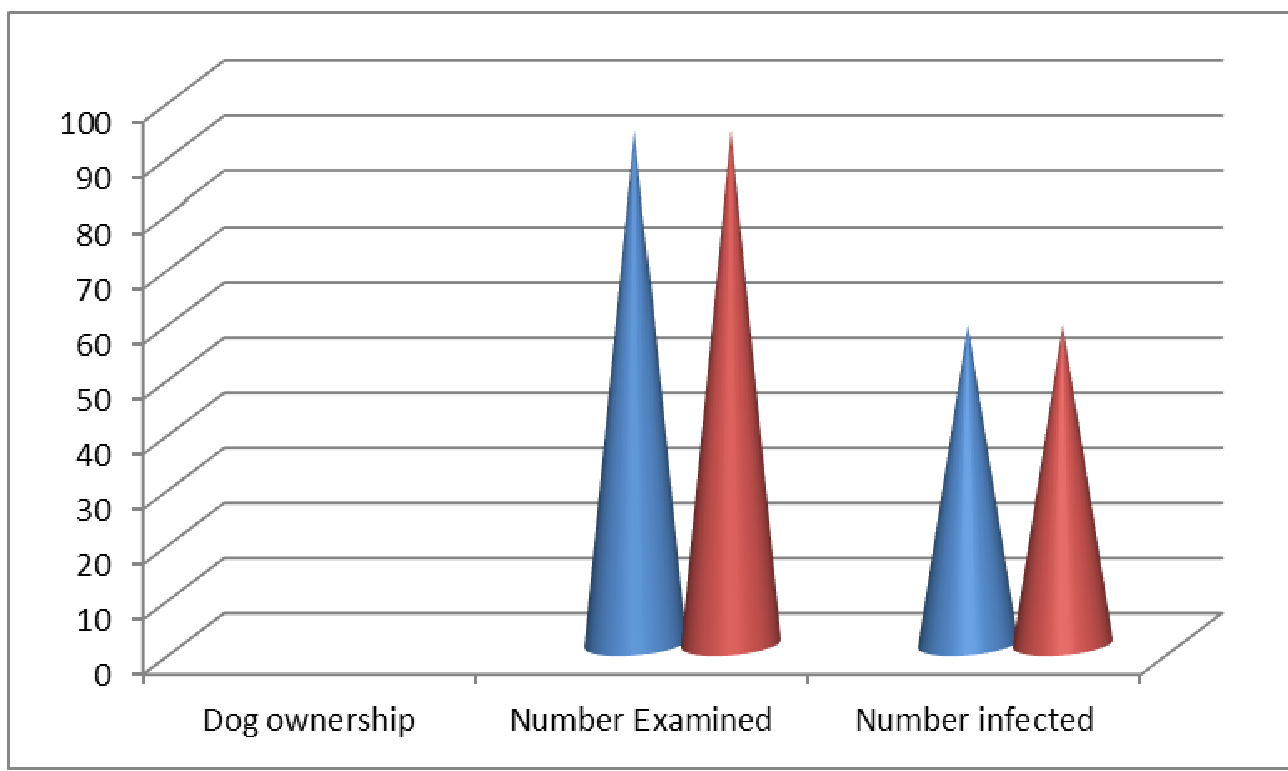

Figure 5. Seroprevalence of Anti-Toxocara Antibody based on Dog ownership

In this study the relationship between dog ownership and Toxocara infection as shown in figure 5 showed a negative correction ( $\mathrm{r}-0.0977)$, and is non-significant $(\mathrm{P}=0.0203)$ signifying that having a dog or not, individuals stand equal chances of contacting Toxocariasis. This finding is contrary to earlier findings reported by Yakubu and Audu (2009), in Vom Plateau State Nigeria. That high seroprevalence rate was found among dog meat eaters than those who do not. Since dogs have also been reported to carry larvae for years in their body (OliveiraSequera, 2002). It is therefore reasonable to speculate that eating raw or uncooked infected dog meat might be associated with human Toxocara canis infection as in the case in dogs that eat infected paratenic hosts. This finds meaning when it is considered that consumption of raw or uncooked meat of other paratenic hosts (such as beef, pork, chicken etc.) leads to the release of T.canis larvae from the muscle to infect humans (Tiara, et al, 2004).

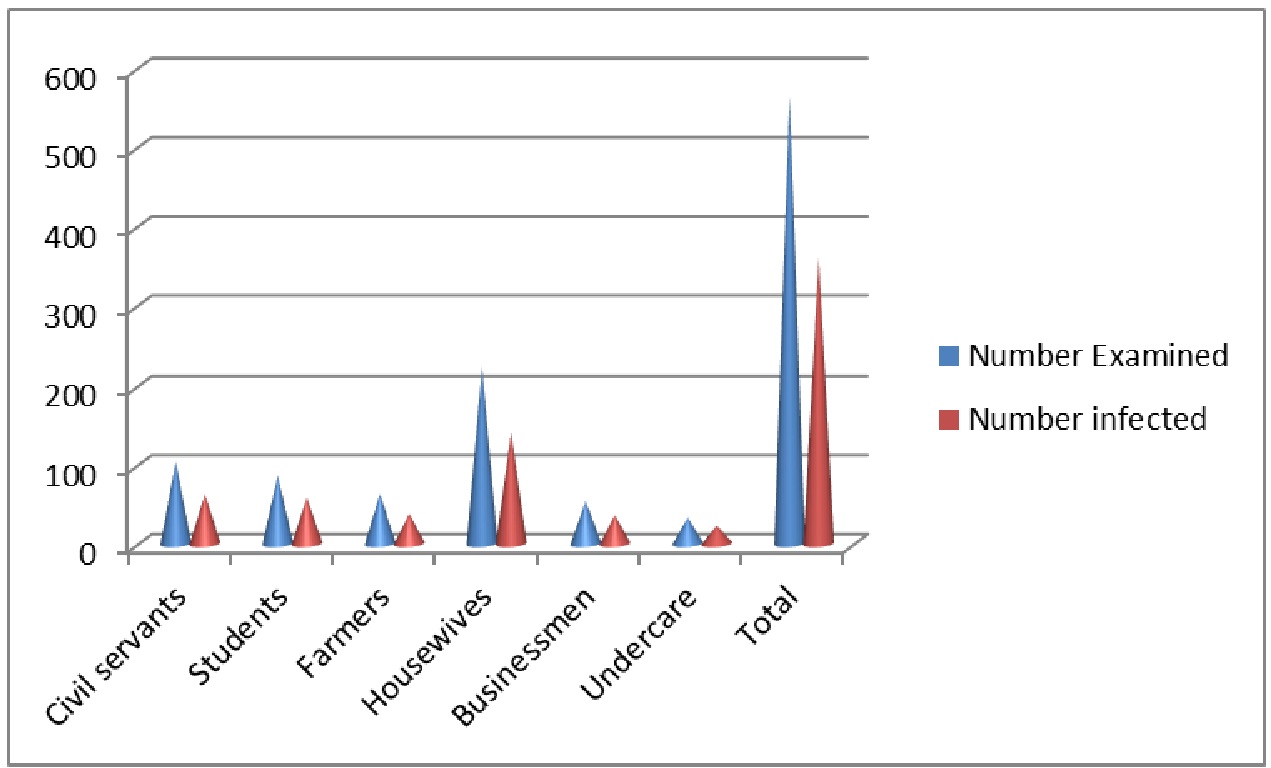

Figure6. Seroprevalence of Anti-Toxocara Antibody titre with Socio-economic status

Finding in this study based on socioeconomic status figure 6 indicates that housewives had 141(25\%) with under care accounting for 23(4.1\%). The probability of housewives having a higher prevalence rate could be attributed to their educational level as most of the housewives examined in the study area have no formal education. This high rate amongst housewives agrees with an earlier work reported by Wolfe and Wright (2003). Where they reported that maternal education and low-socioeconomic conditions have also been related to Toxocara zoonosis. Housewives who are responsible of taking care of their home may be exposed to canine litters. They may also be exposed to the risk of handling embryonated eggs of $T$. canis through handling of paratenic host or through unwashed contaminated fruits and vegetables as reported by Klapec and Borecka (2012). Higher seroprevalence rate as found in housewives in this study agrees with similar work reported by 
Alcantara-Nerves et al,.(2011) and Mendonca et al,. (2012), where they reported that maternal education is highly associated with Toxocariasis infection. Other possible factor responsible for housewives having higher prevalence could be attribute to their role in farming as most housewives in the study area are engaged in farming activities more than their male counterpart. This agrees with findings of Pius et al,. (2012) on the prevalence and risk factors for zoonotic helmith infection among humans and animals in Jos, Nigeria 2005-2009. Being mostly farmers, the housewives are exposed to infection sources which include exposure to animals and soil which serve as reservoir of infection.

Under care (Children under one year) accounted for 23(4.1\%). This low prevalence rate could be attribute to the fact that children within this age are small and not left on their own to be exposed to common environmental risk factors such as playing in the soil or with pet. But as they increase in age, the risk of infection increases as earlier reported by Susana et al,. (2014). This could be attributed to change in behaviour, since children play more often and have closer contact with potentially contaminated soil in yards and sand pits and often put their fingers into their mouths, sometimes even eating dirt's (Good et al,. 2004). Findings in this study that shows that socio-economic of individuals have a low positive correlation $(\mathrm{r}=0.0547)$ and is non significant ( $\mathrm{P}=0.1946)$ to T.canis infection this agrees with an earlier report made by Filho et al,. (2002), in Brazil; which showed a significant effect of Socio-economic parameters on human infection rates with Toxocariasis. Similar findings have also been reported by Tina et al,. (2016).

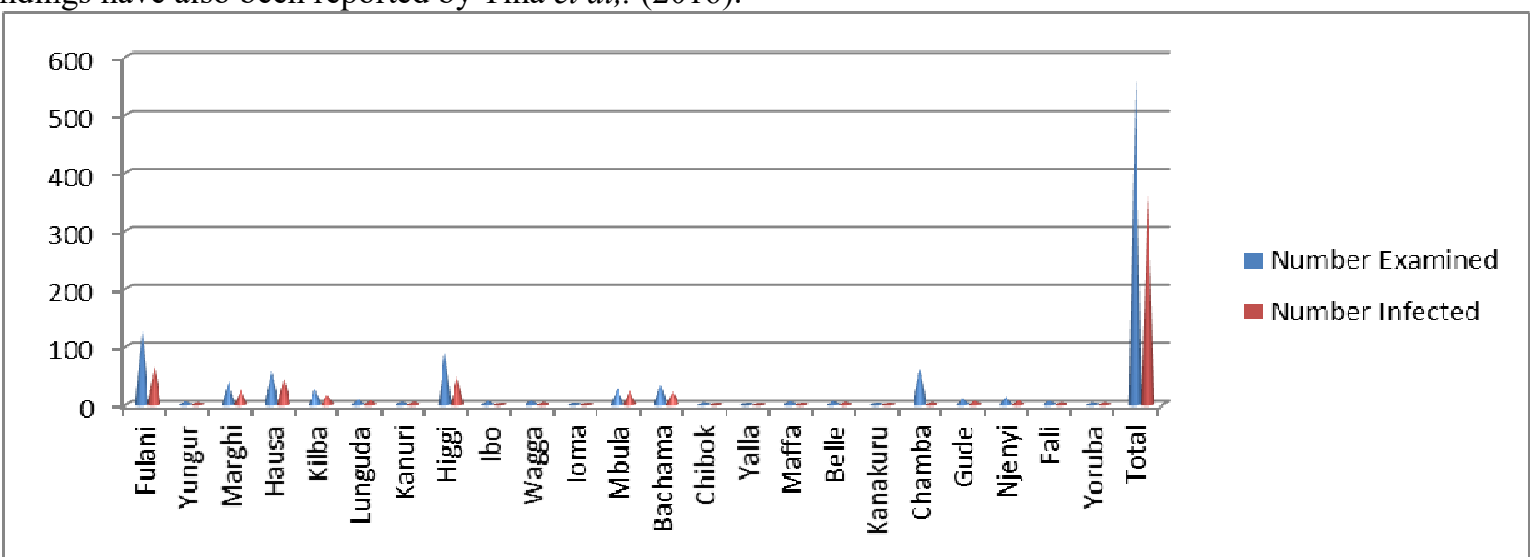

Figure7. Seroprevalence of Toxocara canis Antibody based on Tribe

Results based on Tribes figure 7 reveals that, Fulani had 67(11.88\%), Higgi 48(8.51\%), Chamba 47(8.33\%) and Hausa 45(7.98\%) with other Tribes having lower rates of less than 1\% which includes Fali, Ibo, Chibok and Yoruba. The Fulani's' accounting for a relatively higher rate can be attributed to low level of immunity due to poor nutritional status, poor maternal education, exposure of contaminated soil environment, poor personal hygiene conditions especially lack of washing hands after having contact with contaminated soil and animals. The fact that all the Fulani's' sampled are Moslems and yet had a higher rate, explains the fact that Religion is significant to contacting Toxocariasis. The Higgis' accounting for 48(8.51\%) who are predominantly Christians and mostly keep dogs still support that Religion is a significant factor to Toxocariasis. Activities such as farming amongst Higgis', Chambas' and even among some Hausas' could be said to be a factor that might helped in exposing these Tribes to possible risk factors as outline earlier. These tribes also keep dogs for hunting purposes which also increases their risk of infection. Tribes known to be dog meat eaters in the study area such as the Lungudas' had $8(1.42 \%)$. This findings is contrary to the findings reported by Yakubu and Audu (2009), who reported in a study carried out in Vom Plateau State, Nigeria that eating dog meat is a factor associated with Toxocara infection, like wise involving in dog trade, meat preparation, eating are also factors associate with Toxocara infection. Variations in infection rates based on Tribes showed a low positive correlation to AntiToxocara Antibody $(r=0.0285)$ and non-significant $(\mathrm{P}=0.4994)$ signifying that some tribes may have higher rates than others.

\section{Conclusion}

In conclusion the study showed that there is a very high Seroprevalence of Toxocara canis Antibody titre amongst the studied population

\section{Recommendations}

Therefore it is recommended that Anti-Toxocara Antibody screening be included in the routine medical diagnosis. It is also recommended that further studies on molecular diagnosis be carried out to ascertain the gene markers responsible for the pathology caused to the host by T.canis 


\section{Acknowledgement.}

Authors wish to acknowledge the contributions of the Management, Laboratory Scientist of the Federal Medical Centre Yola, Adamawa State Nigeria. Adamawa State Ministry of Health and the State Hospital Management Board.

\section{References}

Adebayo, A .A and Tukur, A.I. (1999). Adamawa State in map $1^{\text {st }}$ Edition Paraclete publishers Yola. 1-3

Ajayi, O.O.,Duhulinska, D.D., Agwale, M. and Njoku, M (2000). Frequency of Human Toxocariosis in Jos Plateau State, Nigeria. Mem institute Oswaldo Cruz 95(2):147-149

Alcantara-Nerves, N .M., Veiga, R.V., Dattoli, V.C., Fiaccone, R.L., Esquivel, R.., Cruz, A.A. et al (2011).The effect of single and multiple infections on atopy and wheezing children. Journal Allergy Clinical Immunology, 129: 359-367, e351-353

Alonso, J.M., Bojanich, M.V.L., Chamora, M., Gorodher, J.O., (2000). Toxocara seroprevalence in children from Subtropical City in Agentina. Review of Institute of Medicine in Tropics, 42, 235-237.

Anna, K., Kacper, T., Eizbieta, O and Artuk, . (2018). Toxocariasis in Children: Poor hygine habits and contact with dogs is related to longer treatment: Parasitology Research http://doi.org/10.1007/500436-018-5833-7.

Anaruwa, F.F., Chiefs, P.P., Correa, CR., Cameron, E.D., Silvvera, E.P.R., Asranha, J.J.B. and Ribeiro, M.C.S.A.(2002. Human Toxocariasis: A Seroepidemiological survey in the municipality of Capins (sp) Brazil. Revista do Institution de Medeicina Tropica de Sao Paulo, 44 (6):12-18

Aydenizöz-Özkayhan, M. Yağci, B. B and Erat, S. (2008). The investigation of Toxocara canis eggs in coats of different dog breeds as a potential transmission route in human Toxocariosis. Veterinary Parasitology. 152: 94-100

Berenji.(2016). Seroepidiomological Study of Toxocariasis in the owners of dogs. Iran Journal of Parasitology, 11: $265-268$

Chiodo, P., Basualdo, J., Ciamela,L., Pezzani, B., Apezteguia, M., Minvielle, M. (2006). Related factors to Human Toxocariasis in rural community of Agentina. Mem institute Oswaldo Cruz, 101: 397-400

Damian, M. M., Martins, M., Sardinha, J.F., Souza, L.O., Chaves, A and Tavares, M. (2007). Frequencia de anticorpo anti-Toxocara canis Comunidade do Rio Uatuma. no Estado do Amazons. Reviews of Society of Brasil Medicine in Tropic, 40: 661-664

Espinoza, Y.A., Huapaya, P.H., Roldan, W.H., Jimennez, S., Arce,Z., Lopez, E. (2008). Clinical and Serological evidence of Toxocara infection in School Children from Morrope ditrict, Lambayeque, Peru. Review of Institute of Medicine in Tropics, 50:105-105

Filho,F.A., Chieffi, C.R.S., Correa et al. (2002). Human Toxocariasis: a seroepidiomological survey in the municipality of campinas (sp), Brazil. Revista do Instituto de Medica Tropical de Sao Paulo, 44 (6): 303 307

Fisher, M. (2003). Toxocara cati: an underestimated zoonotic agent. Trends Parasitology, 19: 167-170

Gabrielli, S., Tasic-Otasevic, S., Ignjatovic, A., Trenkic-Bozinovic., Momcilovic,S., Cancrini, G. (2017). Seroprevalence and risk factors for Toxocara canis infection in Serbia during 2015. Foodborne Pathologic Diseases, 14: 43-49

Good, B., Hollan, C.V., Taylor, M.R., Larragy, J., Moriarty, P. and O’Regan, M. (2004). Ocular Toxocariasis in School Children. Clinical Infections diseases, 39:173-178

Guangxu, M., Celia, V.H., Tao,W., Andreas,H., Chia-Kwung, F., Rick, M.M.,Peter, J.H., and Robin, B.G. (2018) .Human Toxocariasis. The Lancet, 18: 16-17

Klapec, T. and Borecka, A.(2012). Contamination of vegetables, fruits and soil with geohelminths eggs on organic farm in Poland. Annals of Agricultural and Environmental Medicine, 19: 421-425

Mazur-Melewska, K., Maina, A., Figlerowicz, M., Keninitz, P., Shizewski, W.., Michalak, M.(2012). The influence of age on a clinical presentation of Toxocara spp. Infection in Children. Annual Agricultural Environment of Medicine, 19:233-236

Medonca, L.R., Veiga, R.V., Dattoli, V.C., Figueiredo, C.A., Fiaccone, R. Santos, J., et al. (2012). Toxocara Seropositivity, atopy in wheezing Children living in poor neighbourhood in urban Latin America. PLOS Neglected Tropical Diseases, 11. e1888

Nourian, A.A., Amiri, M.M Ataenan, A Haniloo,S.N., Mosavinaab, and Baali, H. (2008). Seroepidiomological study for Toxocara among children in Annan-Northwest of Iran. Pakistan Journal of Biological Sciences. 11 (14): 1844-1847

Olievira-Sequera, T.C.G., Amarate, A.F.T., FerrariT.B. and Nunes, L.C. (2002). Prevalence of intestinal parasites of dogs in Soa Paulo, Brazil. Veterinary Parasitology, 75: 56-71

Overgaauw, P.A.; Van Zutphen, L.; Hoek, D.; Yaya, F.O.; Roelfsema, J. et al.(2009). Zoonotic parasites in faecal samples and fur from dogs and cats in The Netherlands. Veterinary Parasitology.163, (1-2), 115-122

Pius, S.E., Raymond, J., Ndahi, M.D.,Patrick,N. and Monica, M.(2012). Prevalence and risk factors for zoonotic 
helminth infection among humans and animals- Jos Nigeria, 2005-2009. Pan African Medical Journal, $12: 6$

Roddie, G., Stafford P. Holland.C and Wolfe, A. (2008). Contamination of dog hair with eggs of Toxocara canis. Veterinary Parasitology. 152: 85-93

Romero, N.C., Mendoza, M.G.D., Yanez, A.S.,Ponce, M.M., Bustamante, M.P., Rairez, D.N.(2013). Prevalence and risk factors associated with Toxocara canis infection in children. Scientific World Journal, 1: 572089

Smith, R and Noordin.(2006). Diagnostic limitations and future trends in the serodiagnosis of Human Toxocariasis. Holland, C.V. and Smith, H.V. (Eds.), Toxocara: The Enigmatic Parasite, CABI Publishing, CAB International, Wallingford, Oxfordshire, UK: 89-112

Susana, A., Graciela, I.S., Reinaldo, F., Graciela, C., Lola, B. and Nilda, R. (2014).Toxocariais: Seroprevalence in abandoned- institutionalized children and infants, 46(1):3-6

Taira, K., Saeed, I., Permin, A., Kapel, C.M.O. (2014) Zoonotic risk of Toxocara canis infection through consumption of pig or poultry viscera Veterinary Parasitology. 121: 115-124

Tina, M., Mahani-Oskouei, M., Fallah, E., Safiayan, A and Mahami-Oskouei, L.(2016). Latent an Asymptomatic Toxocara Infection among young population in Northwest Iran: The necessity of informing people as a potential Health Risk. Scientifica. 1-5

Traversa, D.(2012). Pet roundworms and hookworms: a continuing need for global warming. Parasite Vectors 5:91-100

Wis niewska-Liger, M., Wozniakowska-Gesicka, T., Sobolewska-Dryjanska,J., Markiewicz- Jozwiak, A. and Wiezorek, M.(2012). Analysis of the course and treatment of Toxocariosis in children- a long- term observation, Parasitology Research. 110:2363-2371

Wolfe, A and Wright, J.P.(2003).Human Toxocariosis and direct contact with dogs. Veterinary Research 152(14): 419-422.

Yakubu, R.A., Audu, P.A., Ndamis, I.S and Nock, I.H.(2009).Seroprevalence of Human Toxocara canis infection in Vom, Plateau State, Nigeria. Nigerian Journal of Scientific Research, 8: 11-17. 